Article

\title{
Design of IMC Oriented PID Control Law for Typical Thermal Process in Thermal Power Plant
}

\author{
Yin Ke \\ School of control and computer engineering,North China Electric Power University,Beijing, China \\ *Corresponding author: 1151190407@ncepu.edu.cn
}

\begin{abstract}
In the development of automatic control, PID control is the oldest of the basic control mode, the control algorithm is the most widely used in engineering, especially the applied research of robustness has extensive engineering practical value. In this paper, based on the principle of the internal model PID parameter setting method was studied, through different approximate processing method, derived the three different formula of PID internal model setting and simulation verify the effectiveness of the algorithm. At the same time, the robustness of the controlled process under different parameter perturbation is theoretically analyzed and simulated.Finally, the paper summarizes the whole paper and looks forward to the future development trend.
\end{abstract}

Keywords: internal mode control,PID control,robustness,simulation

\section{Introduction}

As a new control method, internal mode control(IMC) was formally proposed by Garica and Morariin[1] in 1982. Rivera[2] first introduced the idea of IMC into the design of PID controller, and established the relationship between the filter parameters and PID controller parameters. Then more and more scholars tried to design PID controller using IMC principle[3][4][5].Compared with classical PID control[6], the structure of the IMC-PID controller was simple, and there was only one setting parameter. With the continuous development and improvement of IMC, the conventional IMC method has been developed to the intelligent control method. Many scholars combined IMC with adaptive control[7][8], fuzzy control[9], neural network[10] and so on, which had certain advantages in the control of complex process. Truong Nquyen Luan Vu and Le Hien Giang[11] had proved that IMC-PID controller can be applied to first-order and time-delay process, and it had certain robustness.Liang Wei-Ping,Ge Xiao-Jing[12] studied a fuzzy algorithm control system based on IMC, which can adjust the proportional coefficient of IMC-PID controller online.in order to improve the system robustness and control performance, Zhi-cheng Zhao[13]andYing -ming Li[14] both presented an adaptive IMC-PID control scheme based on neural network, through neural network self-learning to adjust the parameters of the IMC - PID controller, . In reference[15], the design of a fractional order proportional integral differential controller was proposed. Although satisfactory results were obtained, the output was still undamped and there were many parameters need to be optimized. Bayu Jayawardhana,Hartmut Logemann and Eugene P.Ryan[16] presented a kind of PID control of second-order systems with hysteresis for constant reference signal asymptotic tracking and constant interference signal suppression.But the satisfactory robustness can not be obtained.Zhao and Wang[17] presented that use IMC-PID to control the superheated steam pressure.PID controller is still the most widely used controller in thermal power plant.Alberto Leva[18] introduced the performance and robustness improvement in the IMC-PID tuning method.In addition, the IMC-PID controller can be designed based on different algorithms, such as NPSO algorithm[19],LMI algorithm[20],Novel Bat algorithm[21] and so on.In summary, the IMC-PID 
controller is still widely applicable to solving the current practical process control problems. It can be believed that the application of IMC-PID control will obtain satisfactory control effect in the future.In this paper, the PID control algorithm based on IMC is studied in the process of second order inertia with pure delay. In structure with pure time-delay system in the process of the IMC - PID controller, an inevitable problem is the lag of approximate problem, especially when the pure time lag is large, the first-order Pade approximation can bring big error, so choose which kind of approximate method can obtain better control performance, is the problem to be solved in this paper.

\section{Design of PID controller based on IMC}

\subsection{The basic principle of IMC}

The schematic diagram of IMC is showed in Fig.1.

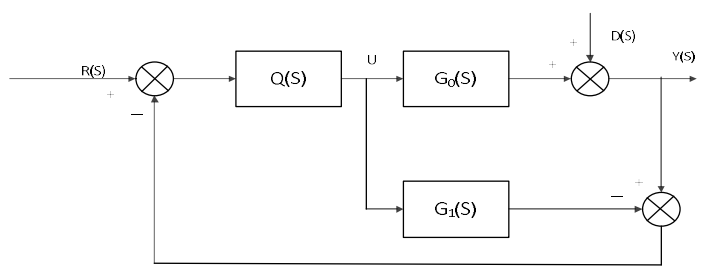

Fig.1 Internal model control schematic diagram

In this figure ,continuous time controller is $\mathrm{Q}(\mathrm{S})$, the controlled process is $\mathrm{G}_{0}$ (s) , the process model is G1(s) which can be decomposed into reversible part $G_{-}(\mathrm{s})$ and irreversible part $G_{+}(s)$, irreversible part includes all the instability of zero and delay. $G 1=G_{-} \times G_{+}$.Controller is set to $\mathrm{Q}(s)=G_{-}^{-1}(\mathrm{~s}) R(s), \mathrm{R}(\mathrm{s})$ is a $\mathrm{n}$-th order low pass filter, $\mathrm{R}(s)=1 /(\lambda s+1)^{n}$.

Need a low-pass filter to make the controller causal, $\lambda$ is the setting parameters of IMC, it have a significant impact on the system performance and robustness. Need to compromise between the speediness and robustness setting, especially in the time-varying delay system.

\subsection{The relationship between IMC controller and classical feedback controller}

The IMC structure of equivalent and principle diagram of conventional PID control system are showed in Fig.2,Fig.3.

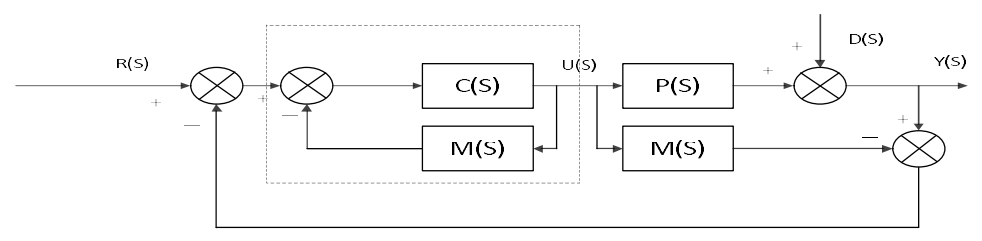

Fig.2 IMC structure of equivalent principle diagram

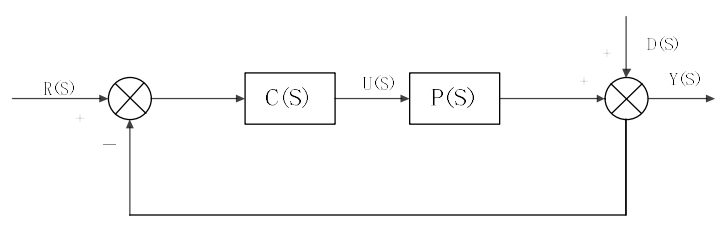

Fig.3 Principle diagram of conventional PID control system 
The internal mode controller $\mathrm{Q}(\mathrm{s})$ is decomposed into the part of the dashed line, and if the input - output relationship shown in Fig. 2 is derived, it will be found that the two models $M$ (s) can be mutually counteracted, and thus the system shown in Fig. 2 can be equivalent to the conventional PID feedback control system shown in Fig. 3, resulting in $C(\mathrm{~s})$ and $\mathrm{Q}$ (s) relationship as follows: $C(s)=\frac{Q(s)}{1-M(s) Q(s)}$.

\subsection{Design step of the IMC-PID controller}

The design ideas of IMC - PID controller is convert the internal mode controller to the solution of the parameter, and then from the angle of the IMC to design the controller. IMC-PID controller design includes four steps: (1) put the model $\mathrm{M}(\mathrm{s})$ into all pass sections $\mathrm{M}+(\mathrm{s})$ and minimum phase $\mathrm{M}-(\mathrm{s}), \mathrm{M}_{+}(\mathrm{s})$ contains the Pure hysteresis stage and the zero points of the right half plane. (2) calculate the internal model controller and feedback controller. (3) converting the feedback controller to the selected PID controller. Use of Q (s), by the various power polynomial coefficients of corresponding principle, the solution can be based on IMC principle of the parameters of the PID controller. (4) setting the filter constant $\lambda$.

\subsection{The PID controller parameter setting based on IMC}

The ideal PID controller has the following form: $\mathrm{G}(\mathrm{s})=\mathrm{Kc}(1+1 / \mathrm{Tis}+\mathrm{Tds})$. In order to get the PID controller, it must be approximately treated, especially the time delay included in G(s). In PID controller parameters setting based on internal model principle, most of the literature for the pure lag processing is currently using Pade approximation, and the achievements are aim at first-order inertia plus pure lag minimum phase systems, thus making the method has more extensively applicable.

In the case that the model is known, the only adjustable parameter of the IMC-PID controller is the filter constant. Therefore, the integral of the IMC-PID controller is the parameter of the tuning filter. So we need a new setting method, which can set the filter constant values for a class of system , and this method can be applied to the second-order inertia plus pure lag system.

The IMC-PID setting step: (1) the low order equivalent (2) model decomposition(3) find the derivative values at $\mathrm{s}=0 ;(4)$ take $\mathrm{r}=\mathrm{l}$, and select the time constant of the filter to find the derivative values of $\mathrm{D}(\mathrm{s})$ at $\mathrm{s}=0$; (5) evaluate the derivatives of $\mathrm{f}(\mathrm{s})$ at $\mathrm{s}=0$; (6) take the PID controller parameters; (7) simulation verification or on-site observation control effect, if satisfied, end; If you are not satisfied about the result, return to step 4 to find a new option. From the above internal model PID setting step, the entire process of setting, only the filter time constant $\lambda$ is need to be adjusted, all the parameters of the controller are related to the filter time constant. With the increase of lambda, Kc and Td will tend to zero, and the integral time Ti would be closer to the TP.

\section{The study of PID control algorithm based on IMC in second order inertial plus pure delay process}

The actual control system from the process model can be divided into two categories, one kind is the first-order inertia plus pure delay process, another kind is the second-order inertia plus pure delay process.So the first thing we should be clear is the system of pure lag link approximation method. Pure lag usually adopts three types of approximate methods, the most commonly used approximate method is first order pade approximation.The approximate formula of the pure hysteresis term: $\mathrm{e}^{-\theta s} \approx(1-0.5 \theta s) /(1+0.5 \theta s)$.Also there are two order pade approximation: $\mathrm{e}^{-\theta s} \approx\left(2-\theta s+0.5 \theta^{2} s^{2}\right) /\left(2+\theta s+0.5 \theta^{2} s^{2}\right) \quad$.The second order asymmetric pade approximation : $\mathrm{e}^{-\theta s} \approx\left(1-0.613 \theta s+0.125 \theta^{2} s^{2}\right) /(1+0.387 \theta s)$. In these three methods, first order pade approximation calculation is simple, and it can be applicable to big lag process that exist in the actual industrial thermal process. 


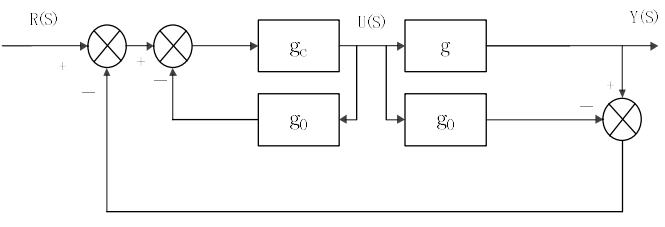

Fig.4 the principle diagram of PID algorithm based on internal model

In this figure,continuous time controller is gc, the controlled process is g,the process model is $\mathrm{g} 0$ which can be decomposed into reversible part $\mathrm{g}_{1}$ and irreversible part $\mathrm{g}_{2}$, irreversible part includes all the instability of zero and delay. $\mathrm{g}_{0}=g_{1} \times g_{2}$. Inter mode controller is set to ${ }^{\mathrm{Q}(s)=g_{1}^{-1} f}, \mathrm{f}(\mathrm{s})$ is a $\mathrm{n}$ order low pass filter, $\mathrm{f}(s)=1 /(\lambda s+1)^{n}$. In general, the controlled process model is assumed to be $\mathrm{g}_{0}(s)=\frac{K}{\left(T_{1} s+1\right)\left(T_{2} s+1\right)} e^{-\tau_{m} s}$.

\subsection{Type I}

The first order pade approximation method is used for the pure hysteresis loop in the model.Take $\mathrm{e}^{-\tau_{m} s} \approx\left(1-0.5 \tau_{m} s\right) /\left(1+0.5 \tau_{m} s\right)$.The order number of the low pass filter is 1 , that is , $\mathrm{n}=1$.The approximate of the controlled process model is : $\mathrm{g}_{0}(s)=\frac{\mathrm{k}}{\left(T_{1} s+1\right)\left(T_{2} s+1\right)} \frac{\left(1-0.5 \tau_{m} s\right)}{\left(1+0.5 \tau_{m} s\right)}$. Decompose the model into $\quad \mathrm{g}_{1}(s)=\frac{K}{\left(T_{1} s+1\right)\left(T_{2} s+1\right)\left(1+0.5 \tau_{m} s\right)} \quad, \quad \mathrm{g}_{2}(s)=1-0.5 \tau_{m} s \quad$.The internal mode controller is $Q\left((\mathrm{~s})=g_{1}^{-1}(s) f(s)\right.$.Feedback controller $\mathrm{G}$ is $\quad: G_{P I D(s)}=\frac{1}{g_{1} f^{-1}-g_{0}}=\frac{1}{g_{1}\left(f^{-1}-g_{2}\right)}=\frac{\left(T_{1} s+1\right)\left(T_{2} s+1\right)}{K\left(\lambda+0.5 \tau_{m}\right) s}\left(1+0.5 \tau_{m} s\right)$ $\approx \frac{1}{K\left(\lambda+0.5 \tau_{\mathrm{m}}\right)}\left(T_{1}+T_{2}+\frac{1}{S}+T_{1} T_{2} S\right)=\frac{T_{1}+T_{2}}{K\left(\lambda+0.5 \tau_{m}\right)}\left[1+\frac{1}{\left(T_{1}+T_{2}\right) s}+\frac{T_{1} T_{2}}{T_{1}+T_{2}} s\right]$. Through $\quad G_{P I D}\left((s)=K_{\mathrm{p}}\left(1+1 / T_{i} s+T_{d} s\right)\right.$, we can know that $K_{\mathrm{p}}=\frac{T_{1}+T_{2}}{K\left(\lambda+0.5 \tau_{\mathrm{m}}\right)}, T_{\mathrm{i}}=T_{1}+T_{2}, T_{d}=\frac{T_{1} T_{2}}{T_{1}+T_{2}}$

\subsection{Type II}

Take $\mathrm{e}^{-\tau_{m} s} \approx\left(1-0.5 \tau_{m} s\right) /\left(1+0.5 \tau_{m} s\right)$, The order number of the low pass filter is 1 , that is , $\mathrm{n}=1$.The approximate of the controlled process model is : $\mathrm{g}_{0}(s)=\frac{\mathrm{k}}{\left(T_{1} S+1\right)\left(T_{2} \mathrm{~s}+1\right)} \frac{\left(1-0.5 \tau_{m} s\right)}{\left(1+0.5 \tau_{m} s\right)}$.Decompose the model into $\mathrm{g}_{1}(s)=\frac{K}{\left(T_{1} s+1\right)\left(T_{2} \mathrm{~s}+1\right)}, \mathrm{g}_{2}(s)=\frac{1-0.5 \tau_{\mathrm{m}} s}{1+0.5 \tau_{m} s}$. The feedback controller $\mathrm{G}$ is $: G_{P I D(s)}=\frac{1}{g_{1} f^{-1}-g_{0}}=\frac{1}{g_{1}\left(f^{-1}-g_{2}\right)}$ $=\frac{\left(T_{1} s+1\right)\left(T_{2} s+1\right)\left(1+0.5 \tau_{m} s\right)}{K\left(\left(\lambda+\tau_{m}\right) s+0.5 \tau_{m} \lambda s^{2}\right)}$ $\approx \frac{1}{K} \frac{T_{1}+T_{2}+0.5 \tau_{m}}{\lambda+\tau_{m}} \frac{1}{\frac{0.5 \tau_{m} \lambda}{\lambda+\tau_{m}} s+1}\left[1+\frac{1}{\left(T_{1}+T_{2}+0.5 \tau_{m}\right) s}+\frac{T_{1} T_{2}+0.5 \tau_{m} T_{1}+0.5 \tau_{\mathrm{m}} T_{2}}{T_{1}+T_{2}+0.5 \tau_{m}} s\right]$.

Through $G_{P I D(s)}=K_{\mathrm{p}}\left(1+1 / T_{i} s+T_{d} s\right)\left(\frac{1}{T_{f}+1}\right), T_{\mathrm{f}}$ is the time constant of first order inertia filter.Then the PID parameters can be obtained. $K_{\mathrm{p}}=\frac{T_{1}+T_{2}+0.5 \tau_{m}}{K\left(\lambda+\tau_{\mathrm{m}}\right)}, T_{\mathrm{i}}=T_{1}+T_{2}+0.5 \tau_{m}, T_{d}=\frac{T_{1} T_{2}+0.5 \tau_{m}\left(T_{1}+T_{2}\right)}{T_{1}+T_{2}+0.5 \tau_{m}}, T_{\mathrm{f}}=\frac{0.5 \tau_{m} \lambda}{\lambda+\tau_{m}}$

\subsection{TypeIII}


Take $\mathrm{e}^{-\tau_{m} s} \approx\left(1-\tau_{m} s\right)$, The order number of the low pass filter is 1 , that is , $\mathrm{n}=1$.The approximate of the controlled process model is : $\mathrm{g}_{0}(s)=\frac{\mathrm{k}}{\left(T_{1} s+1\right)\left(T_{2} S+1\right)}\left(1-\tau_{m} s\right)$.Decompose the model into $\mathrm{g}_{1}(s)=\frac{K}{\left(T_{1} s+1\right)\left(T_{2} s+1\right)}, \mathrm{g}_{2}(s)=1-\tau_{\mathrm{m}} s$. The feedback controller $\mathrm{G}$ is :

$G_{P I D(s)}=\frac{1}{g_{1} f^{-1}-g_{0}}=\frac{1}{g_{1}\left(f^{-1}-g_{2}\right)}=\frac{\left(T_{1} s+1\right)\left(T_{2} \mathrm{~s}+1\right)}{K\left(\lambda+\tau_{m}\right) s}=\frac{1}{K} \frac{\left(T_{1}+T_{2}\right) s+T_{1} T_{2} \mathrm{~s}^{2}+1}{\left(\lambda+\tau_{m}\right) s}=\frac{\left(T_{1}+T_{2}\right)}{K\left(\lambda+\tau_{m}\right)}\left[1+\frac{T_{1} T_{2}}{T_{1}+T_{2}} \mathrm{~s}+\frac{1}{\left(T_{1}+T_{2}\right) \mathrm{s}}\right]$

Through $G_{P I D((s)}=K_{\mathrm{p}}\left(1+1 / T_{i} s+T_{d} s\right)$, Then the PID parameters can be obtained. $K_{\mathrm{p}}=\frac{\left(T_{1}+T_{2}\right)}{K\left(\lambda+\tau_{\mathrm{m}}\right)}, T_{\mathrm{i}}=T_{1}+T_{2}$, $T_{d}=\frac{T_{1} T_{2}}{T_{1}+T_{2}}$

\subsection{Simulation example}

Consider the process transfer function $\mathrm{g}_{0}(s)=\frac{e^{-2 s}}{(s+1)(2 s+1)}$. Among them, $\tau_{\mathrm{m}}=2, \mathrm{~T}_{1}=1, \mathrm{~T}_{2}=2, \mathrm{~K}=1$.

The control system is simulated by Matlab, and then we can obtain the unit step response curve of the system in four different situations. When $\lambda / \tau_{\mathrm{m}}$ equal to $1.2,2,5$ and 8 , the PID parameters obtained by three approximate methods are shown in Table 1.

Table1.the calculation results when $\lambda / \tau$ m takes different values by different approximate method of PID parameter

\begin{tabular}{l|l|l|l|l|l|l|l|l|l|l}
\hline \multirow{2}{*}{$\lambda / \tau_{\mathbf{m}}$} & \multicolumn{3}{|c|}{ TypeI } & \multicolumn{5}{c|}{ TypeII } & \multicolumn{3}{c}{ TypeIII } \\
\cline { 2 - 11 } & $\mathrm{K}_{\mathrm{p}}$ & $\mathrm{K}_{\mathrm{i}}$ & $\mathrm{K}_{\mathrm{d}}$ & $\mathrm{K}_{\mathrm{p}}$ & $\mathrm{K}_{\mathrm{i}}$ & $\mathrm{K}_{\mathrm{d}}$ & $\mathrm{T}_{\mathrm{f}}$ & $\mathrm{K}_{\mathrm{p}}$ & $\mathrm{K}_{\mathrm{i}}$ & $\mathrm{K}_{\mathrm{d}}$ \\
\hline 1.2 & $\begin{array}{l}0.8 \\
823\end{array}$ & $\begin{array}{l}0.2 \\
941\end{array}$ & $\begin{array}{l}0.58 \\
82\end{array}$ & $\begin{array}{l}0.9 \\
090\end{array}$ & $\begin{array}{l}0.22 \\
72\end{array}$ & $\begin{array}{l}1.1 \\
363\end{array}$ & $\begin{array}{l}0.54 \\
54\end{array}$ & 7.5 & 2.5 & 5 \\
\hline 2 & 0.6 & 0.2 & 0.4 & $\begin{array}{l}0.6 \\
666\end{array}$ & $\begin{array}{l}0.16 \\
66\end{array}$ & $\begin{array}{l}0.8 \\
333\end{array}$ & $\begin{array}{l}0.66 \\
66\end{array}$ & 1.5 & 0.5 & 1 \\
\hline 5 & 0.2 & 0.0 & 0.18 & 0.3 & 0.08 & 0.4 & 0.83 & 0.37 & 0.12 & 0.25 \\
& 727 & 909 & 18 & 333 & 33 & 166 & 33 & 5 & 5 & \\
\hline 8 & 0.1 & 0.0 & 0.11 & 0.2 & 0.05 & 0.2 & 0.88 & 0.21 & 0.07 & 0.14 \\
& 764 & 588 & 76 & 222 & 55 & 777 & 88 & 42 & 14 & 28 \\
\hline
\end{tabular}

(1)while $\lambda / \tau_{\mathrm{m}}=1.2$, The simulation comparison of first-order system unit step response curve based on the above three approximation methods is shown in the Fig.5.The system diverges when the third method is adopted. 


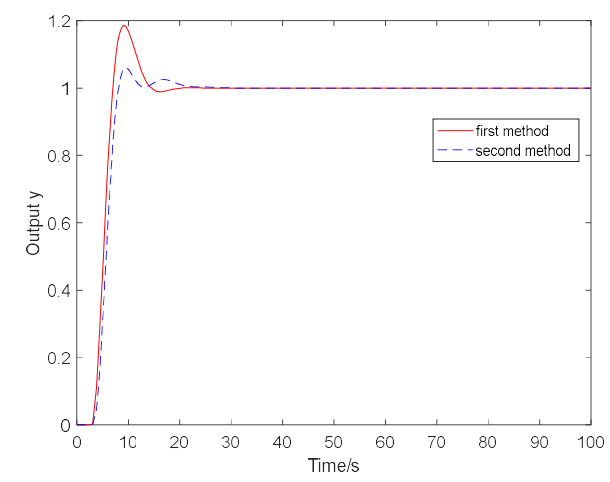

Fig.5 the unit step response curve of the system at $\lambda / \tau_{m}=1.2$

(2)while $\lambda / \tau_{\mathrm{m}}=2$, The simulation comparison of first-order system unit step response curve based on the above three approximation methods is shown in the Fig.6.

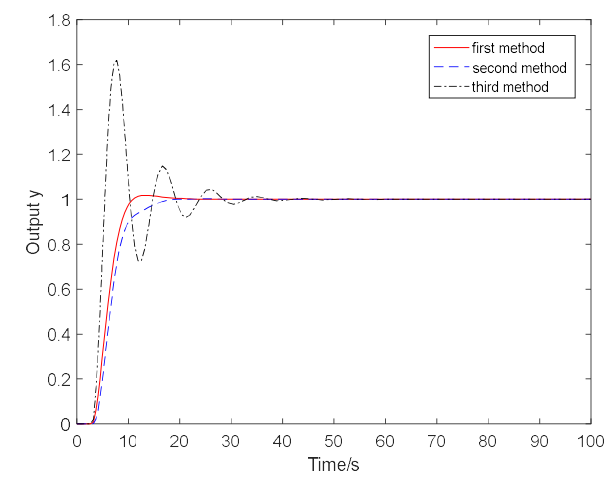

Fig. 6 the unit step response curve of the system at $\lambda / \tau_{\mathrm{m}}=2$

(3)while $\lambda / \tau_{m}=5$, The simulation comparison of first-order system unit step response curve based on the above three approximation methods is shown in the Fig.7.

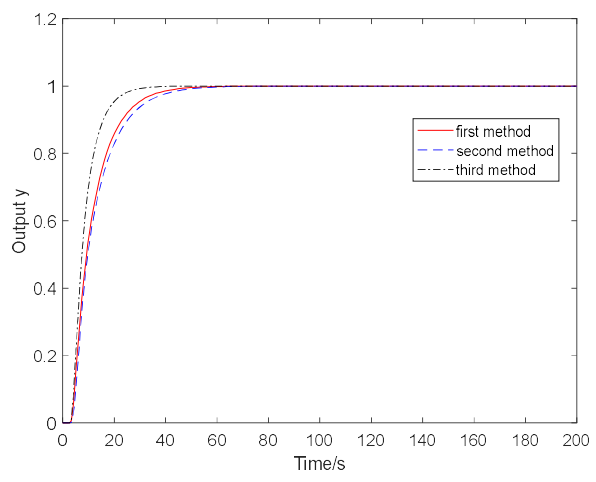

Fig.7 the unit step response curve of the system at $\lambda / \tau_{\mathrm{m}}=5$

(4)while $\lambda / \tau_{\mathrm{m}}=8$, The simulation comparison of first-order system unit step response curve based on the above three approximation methods is shown in the Fig.8. 


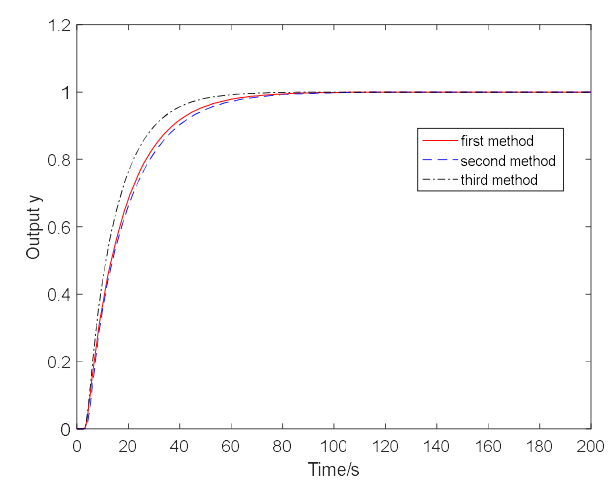

Fig. 8 the unit step response curve of the system at $\lambda / \tau_{\mathrm{m}}=8$

In the following, for the second order controlled process under different parameters, the first method is adopted,take $\lambda / \tau \mathrm{m}=1$.2. The unit step response curve of the system is drawn separately.

(1)The unit step response curve of the system is shown in the Fig.9 when the process gain K equal to $0.5,1,1.5$.

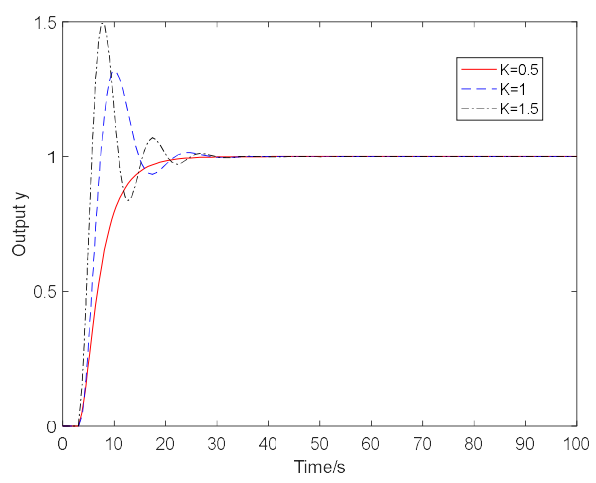

Fig.9 The unit step response curve of the system during the gain $\mathrm{K}$ perturbed

It can be seen from the figure that when the process gain $\mathrm{K}$ perturbed, the second order system with the first approximation method still has good robustness, and satisfactory performance can be achieved.

(2)When the process time constant $\tau_{\mathrm{m}}$ equal to 3,4,5, the unit step response curve of the system is shown in the Fig.10.

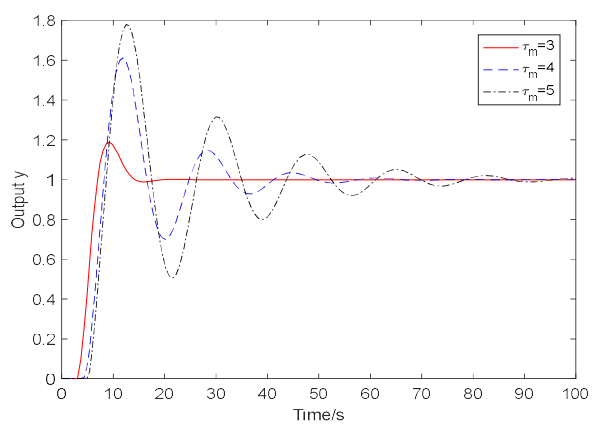


Fig.10 The unit step response curve of the system during the process time constant $\tau \mathrm{m}$ perturbed

It can be seen from the figure that when the process time constant perturbed, the second order system with the first approximation method still has good robustness, and satisfactory performance can be achieved.

(3)When the process inertia time constant $T_{1}$ equal to $0.5,1,2$, the unit step response curve of the system is shown in the Fig. 11.

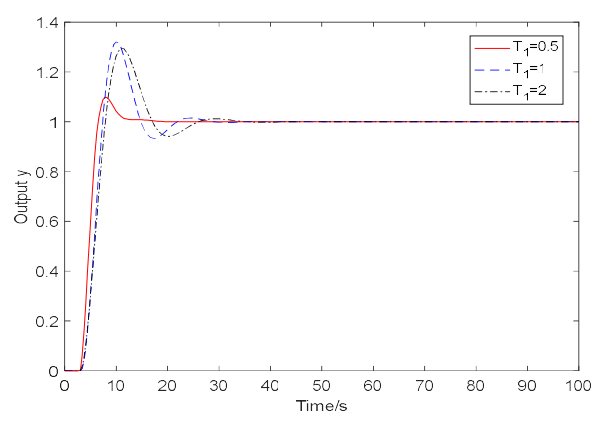

Fig.11 The unit step response curve of the system during the process inertia time constant Tiperturbed

It can be seen from the figure that when the coefficient of the process T1 perturbed, the system with the first approximation method still has good robustness, and satisfactory performance can be achieved.

(4)When the inertia coefficient of the process T2 equal to 1.5,2,2.5, the unit step response curve of the system is shown in the Fig. 12.

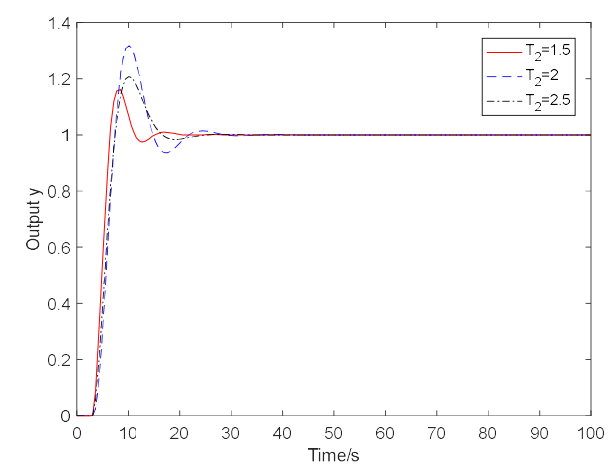

Fig.12 The unit step response curve of the system during the process inertia time constant $\mathrm{T}_{2}$ perturbed

\section{5 summary}

This section in view of the second order controlled process, put forward three different setting of the PID algorithm, respectively the three different methods is presented in the system unit step response curve, and compare their performance index, thus the robustness of them carried on the simulation research. The simulation results show the effectiveness of the proposed algorithm and show that they are robust in parameter perturbation.

\section{Conclusions}


Aiming at the process control object of thermal power plant, this paper makes clear that PID controller is still the most widely used type of controller in thermal power at this stage.But some of the more difficult to control thermal process like the order of inertia plus pure delay, Jane.Single PID control is difficult to achieve satisfactory control effect. In this paper, the IMC is considered as one of the robust control methods. The implementation of this algorithm does not need to transform the existing control system of the thermal power plant, but only in the original PID control system.The process model module is added, namely IMC - PID control.

In this paper, based on the principle of the internal model, PID parameter setting methods are introduced, through different approximate processing methods, deduced the formula of three different PID internal model setting, and simulation verify the effectiveness of the algorithm, at the same time, in view of the controlled process in the case of different parameter perturbation robustness has carried on the theoretical analysis and simulation study. The simulation results verify the validity of the proposed algorithm and show that they are robust to the perturbation of process parameters.

Therefore, it can be concluded that the PID parameter tuning method based on internal model is simple, and the adjustment parameter is single, especially for the improvement of robustness and anti-interference, especially the effect.

However, the analysis of the paper is still very shallow, and the analytical conclusion is still to be further improved, and further theoretical research needs to be carried out. In the future research should be more comprehensive consideration of various factors in actual, strengthening the combination of theory and practice, the IMC - PID controller will be a feasible and effective means of control, a more extensive application and industrial processes.

\section{References}

1. Garcia C E,Morari M.Internal model Control-1.A unifying review and some new results, Ind.Eng.Chem.Process Des.Dev.,1982,21:308-323.

2. Riversa,MorariM,Skogestads.Internalmodelcontrol-4.PID controller design. Ind. Eng. Chem.Process Des.Dev.,1986,25(1):252-265.

3. Li X. F., Wang, Y.G. IMC-PID Controller Design for TITO Process Using Relay Feedback Identification Method. 2014 IEEE -SMC, pp. 3541 - 3546.

4. D.B. Santosh Kumar, R. Padma Sree. Tuning of IMC based PID controllers for integrating systems with time delay. ISA Transactions, vol. 63, pp. 242-255, 2016.

5. MaamarBettayeb. IMC-PID-fractional-order-filter controllers design for integer order systems. ISA Transactions, vol. 53, no. 5, pp.1620-1628, 2013.

6. Davood M. Souran, Mahdi Mir, Armin Mebrabian, Behrooz Razeghi, Majid Hatamian, S. Sina Sebtahmadi.A performance comparison of classical PID, Type-1 and Type-2 fuzzy controller in a three tank level control system. Robotics and Manufacturing Automation , 2014 IEEE International Symposium(Kuala Lumpur,Malaysia).

7. Yasuki Kansha,Li Jia,Min-Sen Chiu.Adaptive IMC controller design using linear multiple models.Journal of the Taiwan Institute of Chemical Engineers.Vol. 41, Issue 4, pp. 446-452,2010.

8. Chih-WeiChu, B.Erik Ydstie, Nikolaos V.Sahinidis.Optimization of IMC-PID Tuning Parameters for Adaptive Control: Part 1.Computer Aided Chemical Engineering,vol.29,pp.758-762,2011.

9. Yuzhong Wang,Qinbing Jin,Ridong Zhang.Improved fuzzy PID controller design using predictive functional control structure.ISA Transactions,vol. 71, pp. 354-363,2017.

10. Z. Hajduk. High accuracy FPGA activation function implementation for neural networks. Neurocomputing, 247 (2017), pp. 59-61. 
11. Truong Nguyen Luan $\mathrm{Vu}$, Le Hieu Giang,Le Linh.Advanced IMC-PID controller design for the disturbance rejection of first order plus time delay processes.2017 System Science and Engineering(Ho Chi Minh City,Vietnam).

12. Liang Wei-Ping,Ge Xiao-Jing.A simulation of fuzzy control algorithm based on the IMC-PID controller.2015 Control Conference(Hang Zhou,China).

13. Zhi cheng Zhao,Jiang gang Zhang,Ming dong Hou.An adaptive IMC-PID control scheme based on neural network. 2009 Industrial Electronics and Applications(Xi'an,China).

14. Ying-ming Li, Ming-dong Hou, Jie-ping Hu. An adaptive IMC-PID control scheme based on neural networks. 2009 Intelligent Systems(Xiamen,China).

15. Ibnu Masngut,Gilang Nugraha Putu Pratama,Adha Imam Cahyadi.Design of fractional-order proportional-integral-derivative controller: Hardware realization. 2018 Information and Communications Technology(Yogyakarta,Indonesia).

16. Bayu Jayawardhana,Hartmut Logemann,Eugene P.Ryan.PID control of second-order systems with hysteresis. The 46th IEEE Conference on Decision and Control, 2007(New Orleans,LA,USA).

17. Zhao Kun-Long,Wang Zai-Ying.Application of boiler superheated steam pressure control which based on IMC-PID.The 27th Control and Decision Conference, 2015 (Qingdao,China).

18. Alberto Leva.Performance and Robustness Improvement in the IMC-PID Tuning Method.European Journal of Control,volume 12,issue 2,pp.195-204,2006.

19. Q.B.Jin,F.Hao,Q.Wang. A multivariable IMC-PID method for non-square large time delay systems using NPSO algorithm.Journal of Process Control,volume 23,issue 5,pp.649-663,2013.

20. Hamideh Najafizadegan, Farshad Merrikh-Bayat, Abolfazl Jalilvand. IMC-PID controller design based on loop shaping via LMI approach.Chemical Engineering Research and Design,vol.124,pp.170-180,2017.

21. S.K.Lakshmanaprabu,U Sabura Banu, P.R.Hemavathy.Fractional order IMC based PID controller design using Novel Bat optimization algorithm for TITO Process.Energy Procedia,vol.117,pp.11251133,2017 . 